\title{
Structure and Function Relationship of Human Heart from DENSE MRI
}

\author{
Abbas N. Moghaddam ${ }^{1,2}$ and Morteza Gharib ${ }^{1}$ \\ ${ }^{1}$ California Institute of Technology, Pasadena, California, USA \\ ${ }^{2}$ David Geffen School of Medicine at UCLA, Los Angeles, California, USA
}

\begin{abstract}
The study here, suggests a macroscopic structure for the Left Ventricle (LV), based on the heart kinematics which is obtained through imaging. The measurement of the heart muscle deformation using the Displacement ENcoding with Stimulated Echoes (DENSE) MRI, which describes the heart kinematics in the Lagrangian frame work, is used to determine the high resolution patterns of true myocardial strain. Subsequently, the tangential Shortening Index (SI) and the thickening of the LV wall are calculated for each data point. Considering the heart as a positive-displacement pump, the contribution of each segment of LV in the heart function, can be determined by the SI and thickening of the wall in the same portion. Hence the SI isosurfaces show the extent and spatial distribution of the heart activity and reveals its macro structure. The structure and function of the heart are, therefore, related which in turn results in a macroscopic model for the LV. In particular, it was observed that the heart functionality is not uniformly distributed in the LV, and the regions with greater effect on the pumping process, form a band which wraps around the heart. These results, which are supported by the established histological evidence, may be considered as a landmark in connecting the structure and function of the heart through imaging. Furthermore, the compatibility of this model with microscopic observations about the fiber direction is investigated [1]. This method may be used for planning as well as post evaluation of the ventriculoplasty.
\end{abstract}

Keywords: cardiac mechanics, image-based biomechanical models, strain, structure, function, tissue deformation.

\section{INTRODUCTION}

Effective modeling of the Left Ventricle (LV) has a significant impact on the evaluation of the heart defects as well as its treatments. Heart function may be investigated and modeled at the molecular level by studying the actin-myosin proteins in the muscle fibers [2,3]. Microscopic modeling of the heart based on the fiber contraction, however, is complicated and consists of considerable simplifications in the distribution and direction of fibers as well as their contractile model. Although each fiber generates a force tangent to itself, at any given location the direction of the resultant force is not known since there is a transmural variation in the fiber directions [4]. This effect can be illustrated by considering a rope when it is pulled along its length. The comprising fibers in the rope are not necessarily aligned in the axial (pulling) direction.

In a similar fashion, we propose to look for macro-structure in the myocardium based on its role as a pathway for the transmission of contractile force. If such a structure exists, knowledge of its normal morphology and function may open a new horizon for better diagnosis strategies, evaluation, modeling, and treatment of heart disease. The goal of this research is to investigate the existence of such a structure through the imaging of the heart kinematics.

We define the dominant macro-structure of the myocardium as the pathway that is determined based on the local contractile behavior of the myocardium. The first required step in determining the heart's macro-structure is to define the regional contractile functionality as oppose to the global function of the heart. In other words, the contribution of each region of the heart in the entire pumping process should be characterized by some measurable factor. The next step is to calculate this characteristic factor for all points in the ventricle wall and discover locations with greater contribution to the overall functionality. The effectiveness of this approach depends on the proper identification of the cardiac regions that exhibit higher contractile activity and the nature of their linkage and inner-connectivity.

Medical Imaging 2007: Physiology, Function, and Structure from Medical Images, edited by Armando Manduca, Xiaoping P. Hu, Proc. of SPIE Vol. 6511, 65111L, (2007) · 1605-7422/07/\$18 · doi: 10.1117/12.711269 


\section{THEORY}

\subsection{Wall thickening}

The regional functionality of the LV is important for the evaluation of dysfunctional myocardial regions. In this respect a regional Ejection Fraction (EF) can be defined by calculating the myocardium area in the short axis images of the LV at the end of systole and diastole [5]. For this approach to work, the LV needs to be segmented and the center axis of the LV tracked during the cardiac cycle. Tracking the center axis of the LV is challenging in this method.

Wall Thickening (WT) and Wall Motion (WM) are two other representative factors for evaluating the LV regional activity [6]. The WT has shown some advantages over WM in applications such as the evaluation of postoperative cardiac function [7].

During the last twenty years, MRI has been used to determine both WT and WM [8], but the early applications were mainly based on the measurements over CINE magnitude images and were not accurate since CINE images only show the epi and endocardium. When MR strain imaging methods such as tagging were developed, however, the local strain analysis showed its superiority to the previous planar WT analysis in the differentiation of normal and dyskinetic regions [9]. Furthermore, these new techniques also improved WT measurement methods. Using tagging along with more precise measures of the WT, Moore et al. showed that WT and some of the contraction indices had better accuracy and tighter normal ranges than the calculated strain components [10].

In this study, using DENSE MRI as the main imaging method, we extended Moore et al. method to show the transmural changes of WT across the wall. We then used the map of WT and a related index, i.e. Shortening Index (SI), to find the macro-structure of the LV.

\subsection{Importance of WT \& SI}

The adult heart is a positive displacement pump which maintains a nearly constant external volume during contraction. The EF as the ratio of the stroke volume to the end diastolic volume, is almost $60 \%$ in a normal human LV [11]. Nevertheless, the contraction of myofibers falls in the range of 12 to $15 \%$. If this contraction only generated a longitudinal shortening in the LV, as shown schematically in Fig. 1(b) using a prolate spheroidal coordinate system, then it would result in an EF of about $15 \%$. This reveals the importance of the wall thickening, as in this schematic a WT of $22 \%$ guarantees not only the incompressibility of the myocardium muscle but also a realistic EF of about $60 \%$ (Fig. 1(c)). These numbers are only quoted to give an idea of the importance of the WT. In a real physiological case,

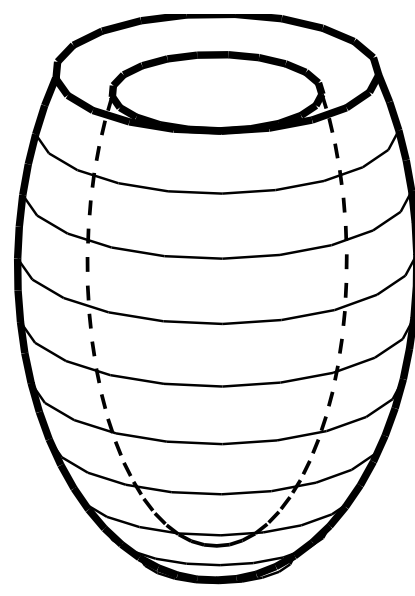

(a)

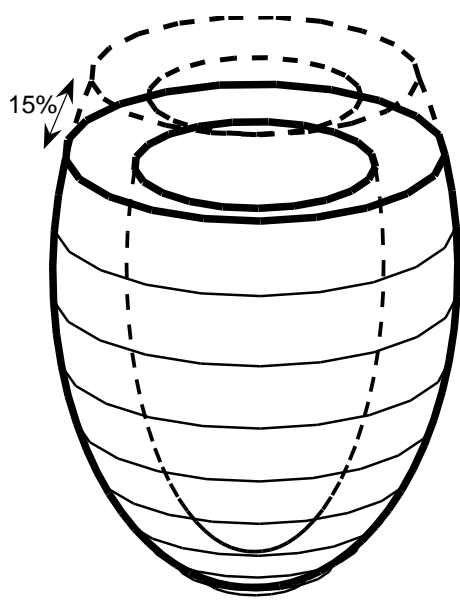

(b)

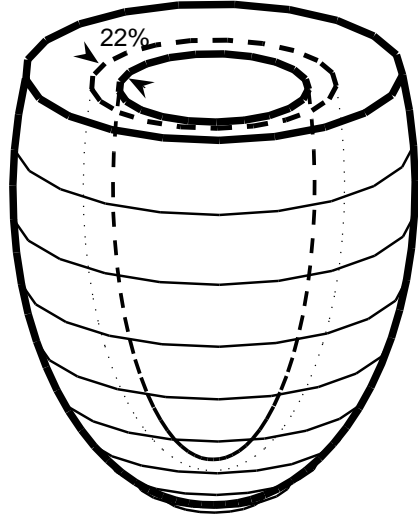

(c)

Figure 1. The model of the LV (a) at end diastole, (b) with only longitudinal contraction and (c) with wall thickening in addition to shortening. 

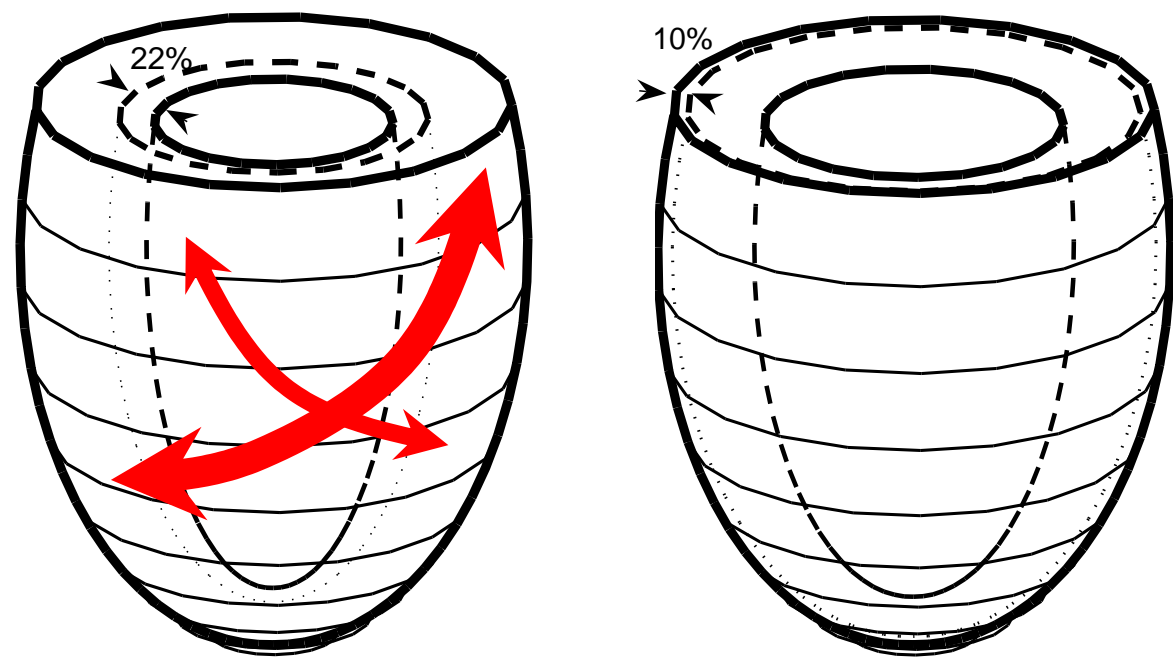

Figure 2. The conservation of mass may be satisfied in many different ways. In this schematic contraction in the endocardium (left) has been compared to the dilation of the epicardium (right). The amount of WT is quite different in these two cases.

the typical thickening of the heart wall is usually higher than $40 \%$ [10].

It is important to note that the wall thickening is not determined solely by the incompressibility of the myocardium. In the schematic of Fig 1, if the LV could dilate circumferentially, a WT of $10 \%$ would satisfy the incompressibility but results in only $15 \%$ EF (Fig. 2). This demonstrates how crucial the circumferential lengthening could be to the heart's operation. The experimental results also reinforce this logic. Fig. 3 illustrates the longitudinal as well as circumferential tensile strains in a long axis slice of the heart at end systole. It shows that at end systole, all points along the wall are contracted circumferentially as well as longitudinally. Therefore, the macroscopic structure should be in the form of two intersecting bands to ensure the existence of surface tension in the LV and create a contraction in both directions. A spiral pathway is the most probable way to unify these intersecting bands. Thus, we may expect the main axes of contraction to be in the form of arrows shown in the schematic of Fig. 2. There is a good evidence for these logical conjectures from MR strain measurements. Moore et al. have shown that the principal strains of the LV wall during the shortening phase are directed in these two speculated directions [10]. At the microstructure level, the surface tension is guaranteed by transmural changes of the fiber directions from left-hand twist at the epicardium to right-hand twist at the endocardium [4]. Recent statistical investigations through 3D diffusion-weighted MRI have shown that in the anisotropic regions of the LV, fibers have two consistent and dominant orientations, approximately $45^{\circ}$ and $117^{\circ}$ [12]. These results, which are supported by previous histological studies[13], may connect the microstructure with the suggested macro-structure.

Moore et al. have suggested a scalar quantity to measure the surface tension in a manner which is not sensitive to the inplane deformation [10]. This quantity, known as the Shortening Index (SI), measures the change of the in-plane area based on the stretch tensor $U$, in the Radial, Longitudinal and Circumferential $(R L C)$ coordinate system, is calculated as:

$$
S I=\sqrt{\left|\left(U_{R C}, U_{C C}, U_{L C}\right) \times\left(U_{R L}, U_{C L}, U_{L L}\right)\right|}-1 .
$$

Furthermore they showed that, considering the incompressibility of the myocardium, the thickening of the wall, WT, is also calculated by elements of $U$ :

$$
W T=\frac{1+U_{R C}\left(U_{C R} U_{L L}-U_{C L} U_{L R}\right)-U_{R L}\left(U_{C R} U_{L C}-U_{C C} U_{L R}\right)}{U_{C C} U_{L L}-U_{C L} U_{L C}}-1,
$$

where $W T$ and $S I$ can be measured at any point inside the heart muscle. 

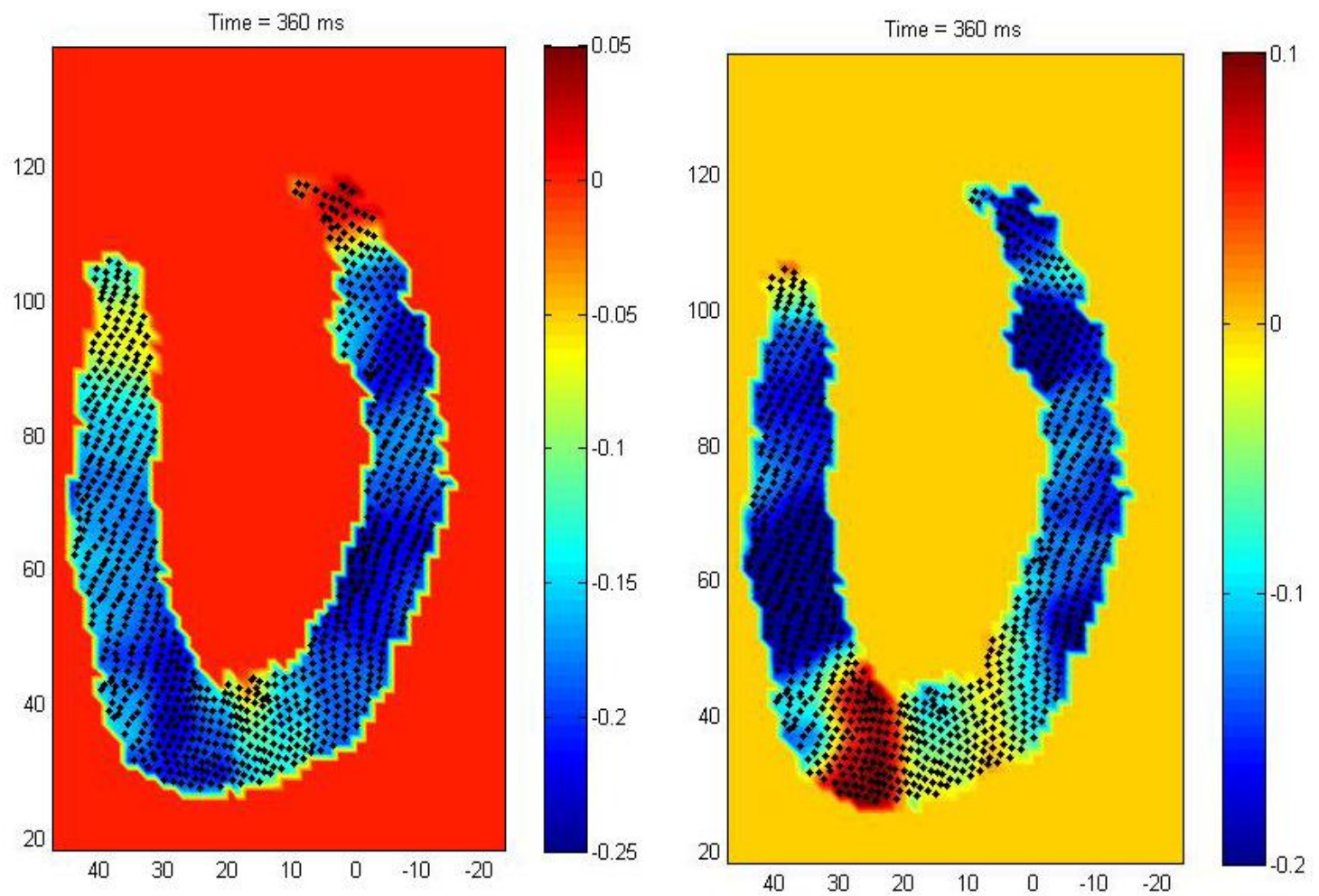

Figure 3. Longitudinal (left) and Circumferential (right) tensile strains at end systole in a normal human LV. The values near the apex are not reliable because of the ambiguity of RLC unit vectors at this region.

Both tensile strains are negative.

Considering that the functionality of the LV comes from wall thickening and its tangential surface tension, these two dependent scalar quantities can be used as the quantitative characteristics for the LV's regional functionality. We calculated the maps of $W T$ and $S I$ with high spatial resolution based on the maximum deformation of the LV, i.e. from the end-diastole to the end-systole. The macro-structure of the LV was subsequently sought by calculation and comparison of the iso-surfaces of these quantities for their relatively higher absolute values.

\section{EXPERIMENTAL METHODS}

DENSE MRI has been used to measure the displacements of the heart muscle at discrete points. For this study, particle tracking was performed on eight short axis (SA) images captured from the heart of a 26 year-old male volunteer. Experiments were performed in a Siemens Trio 3T MR whole body scanner at the Caltech Brain Imaging Center with an eight-channel cardiac array coil. The parameters are; $\mathrm{TR}=3.1 \mathrm{~ms}, \mathrm{TM}=250 \mathrm{~ms}$, number of averages $=3$, number of phases $=2$, in-plane resolution $=1.7 \mathrm{~mm} \times 1.7 \mathrm{~mm}$. Slice thickness of the SA images is $6 \mathrm{~mm}$, while they were spaced every $9 \mathrm{~mm}$. The experiment was performed during free respiration. To synchronize the RF pulses with the motion of the heart and chest, respiratory and cardiac monitoring were achieved using a pneumatic bellows and ECG. The displacement vector field was smoothed by a moving average filtering. We captured the positions of the designated muscle volume elements at the end-diastole and maximum systole in the heart cardiac cycle. 


\section{RESULTS}

The measured displacement field of the myocardium particles is shown in Fig. 4. These displacement fields may be used for calculation of the global, as well as local, quantitative factors of the LV like EF, torsion, and strain maps. Due to the scope of this study, we focus on the $S I$ and $W T$ defined previously. Using the methods described elsewhere (Humphrey, 2005), we calculated the deformation gradient tensor $F$, the right stretch tensor $U$ in the $R L C$ coordinate system, and finally $S I$ and $W T$ using equations (1) \& (2). Having these values for all imaged pixels, their volumetric isosurfaces then were calculated using Matlab software.

For visualization purposes, we set a threshold for the absolute value of these quantities, such that material points with $S I$ (or $W T$ ) below that threshold were removed from the solution domain. We increased this threshold uniformly until the disconnection of the remaining regions started. Fig. 5 shows the results of this procedure for the calculated SI. We observed from the analysis of the dataset acquired from the human subject that contraction strength is not uniformly distributed in the LV, rather it is wrapped around the heart like a band. This band starts from the superior-anterior section of the LV, goes down to its inferior posterior through the LV free wall, rotates around the apex and finally arrives at the septum. The other side of the contraction band initiates from the superior-posterior of the heart and through the LV free wall reaches the septum and becomes connected to the other part.

\section{DISCUSSION}

The resulted macro-structure agrees with our expectations of an intersecting spiral macro structure of the LV. It is important to note that the contraction and WT, as well as the tangential shortening of the wall, happened all around the $\mathrm{LV}$, however our findings show that this activity was not uniformly distributed around the wall. Instead, regions of high contractility were distributed as if a band has been wrapped around the heart, acting as the backbone of the heart contraction.

It is interesting to note that this discovery has a strong histological evidence, i.e., the blunt dissection of the heart in the form of a muscle band by Torrent Guasp [14-16]. He had hypothesized

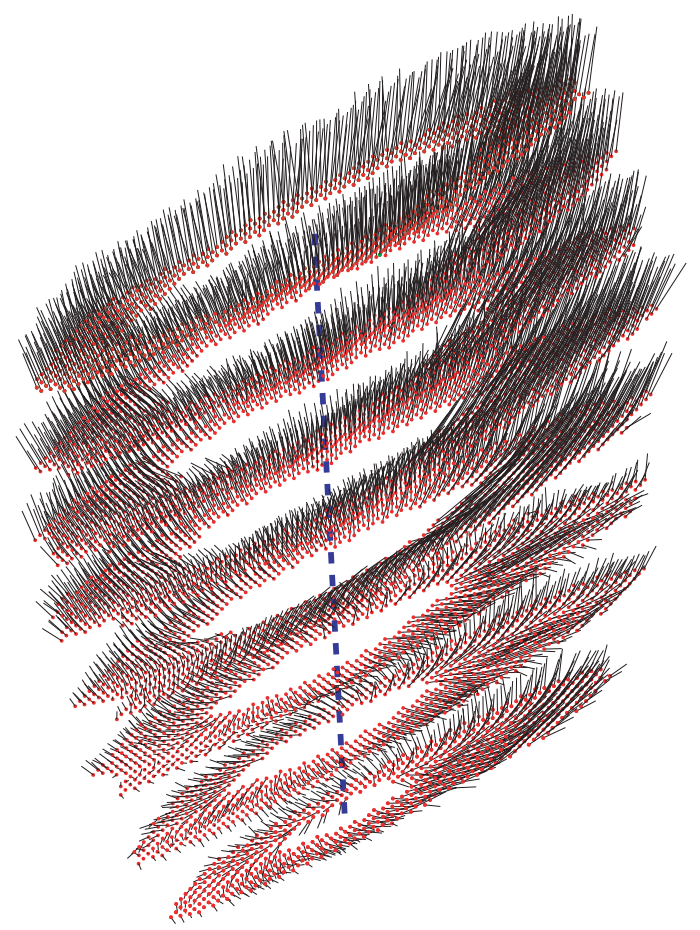

Figure 4. Displacement field of the left ventricle for another heart (case two). The SA slices are not perpendicular to the main axis of the heart (dashed line).

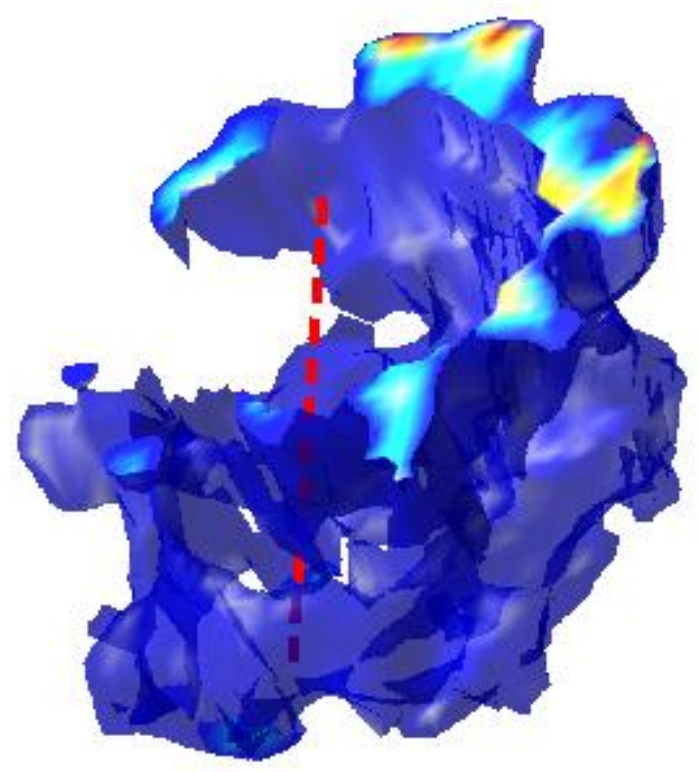

Figure 5. Calculated thresholded isosurfaces of SI for the a healthy volunteer. The path of the muscle band, predicted by Torrent-Guasp theory has a good correlation with the isosurfaces.. 
that the pathway of contraction in the heart is along a muscle band that has the shape of a helical structure. This is the first time that functional data from a beating heart revealed a similar pathway based on maximum local contractility. The most important challenge then is to explain how such a macroscopic structure adheres to the observed micro-pattern of myofibers.

It is important to show that our observation is compatible with myofiber patterns that has been observed through histology [17] and diffusion tensor MRI [12]. The main point of these studies is that the fiber angles change gradually from left-hand twist at the epicardium to right-hand twist at the endocardium. Using the general information of the fiber orientation in the LV wall [17], some previous investigators quantified the fiber orientation by two angles at each point: the helix fiber angle and the transverse angle. The former is defined as the angle between the circumferential direction and the projection of fiber path on the tangential surface while the latter is the angle between the same circumferential direction and the projection of fiber path on the axial cross section of the $\mathrm{LV}[1]$.

Vendelin et. al. generalized the findings of the Streeter by applying it to an axisymmetric case. Furthermore, they simplified the transmural changes of the fiber direction by assuming that the helix fiber angle linearly changes from a positive angle (right-hand twist) sub-endocardially to a negative angle (left-hand twist) sub-epicardially. They also assumed that the transverse angle is a linear function of theta $(\bar{\theta})$, the parameter determining the longitudinal location on the ellipsoid coordinate system, and is tangent to the wall at the endo- and epicardium [1]:

$$
\begin{gathered}
\alpha_{h}=P_{1}+P_{2} \bar{\xi} \\
\alpha_{t}=P_{3} \bar{\theta}\left(1-\bar{\xi}^{2}\right)
\end{gathered}
$$

where $\bar{\xi}$ is the normalized factor in the range of $[-1,1]$ which determines the radial position from endo- to epicardium. They used optimization algorithms to find parameters $P_{1}$ to $P_{3}$ that give the most uniform strain and stress in the myocardium. Using these values, i.e. $P_{1}=22.5, P_{2}=-69, P_{3}=5$, we calculated the path of the fibers. Figure 6 shows a bundle of these fibers that conform with the designated parameters. It illustrates how this bundle can make a muscle band with a configuration similar to the band in Figure 5. If one of these possible bundles shows a slightly denser population of fibers, that collection of muscles will show more contractility. Therefore, higher SI and $W T$ values will be measured over that bundle. Considering the non-symmetrical geometry of the whole heart, it is obvious that the axisymmetry condition can not be quite true for the LV. The other evidence of LV non-symmetry is provided by the tracking the stages of the heart development, that is while the formation of the LV starts with no symmetry there should be some fingerprint of non-symmetry in the grown heart. Thus, the nonuniform density of the fibers is quite expected, which means the contributions of different myocardial regions in the pumping of blood are not equal. 


\section{CONCLUSION}

The relationship of the form and function of the LV has been studied through investigating the spatial distribution of its regional functionality. SI and WT were chosen as the characteristic parameters which show this functionality. These parameters were calculated using DENSE MRI, a technique for measuring the myocardium displacement. We showed how regions with a higher degree of functionality may form a macro-structure for the LV. The histological evidences emphasize the existence of such a band-shaped structure. We further showed the compatibility of the helical-band macro-structure with the micro-structural organization of the fibers in the LV. 


\section{REFERENCES}

[1] M. Vendelin, P. H. Bovendeerd, J. Engelbrecht, and T. Arts, "Optimizing ventricular fibers: uniform strain or stress, but not ATP consumption, leads to high efficiency," Am J Physiol Heart Circ Physiol, vol. 283, pp. H1072-81, Sep 2002.

[2] J. S. Davis, S. Hassanzadeh, S. Winitsky, H. Lin, C. Satorius, R. Vemuri, A. H. Aletras, H. Wen, and N. D. Epstein, "The overall pattern of cardiac contraction depends on a spatial gradient of myosin regulatory light chain phosphorylation," Cell, vol. 107, pp. 631-41, Nov 302001.

[3] J. S. Davis, S. Hassanzadeh, S. Winitsky, H. Wen, A. Aletras, and N. D. Epstein, "A gradient of myosin regulatory light-chain phosphorylation across the ventricular wall supports cardiac torsion," Cold Spring Harb Symp Quant Biol, vol. 67, pp. 345-52, 2002.

[4] D. D. Streeter, Jr., H. M. Spotnitz, D. P. Patel, J. Ross, Jr., and E. H. Sonnenblick, "Fiber orientation in the canine left ventricle during diastole and systole," Circ Res, vol. 24, pp. 339-47, Mar 1969.

[5] A. C. Lapeyre, 3rd, E. Klodas, P. J. Rogers, L. J. Sinak, T. C. Hammell, M. K. O'Connor, and R. J. Gibbons, "Quantitation of regional ejection fractions using gated tomographic imaging with 99mTc-sestamibi," Chest, vol. 127, pp. 778-86, Mar 2005.

[6] H. Fujii, H. Ohashi, Y. Tsutsumi, T. Kawai, K. Iino, and M. Onaka, "Reverse remodeling after endoventricular circular patch plasty in the mid-term period," Interactive CardioVascular and Thoracic Surgery, vol. 4, pp. 602-605, December 1, 20052005.

[7] J. Taki, T. Higuchi, K. Nakajima, I. Matsunari, E. H. Hwang, H. Bunko, M. Kawasuji, G. Watanabe, and N. Tonami, "Electrocardiographic gated (99m)Tc-MIBI SPECT for functional assessment of patients after coronary artery bypass surgery: comparison of wall thickening and wall motion analysis," J Nucl Med, vol. 43, pp. 589-95, May 2002.

[8] U. Sechtem, B. A. Sommerhoff, W. Markiewicz, R. D. White, M. D. Cheitlin, and C. B. Higgins, "Regional left ventricular wall thickening by magnetic resonance imaging: evaluation in normal persons and patients with global and regional dysfunction," Am J Cardiol, vol. 59, pp. 145-51, Jan 11987.

[9] M. J. Gotte, A. C. van Rossum, J. W. R. Twisk, J. P. A. Kuijer, J. T. Marcus, and C. A. Visser, "Quantification of regional contractile function after infarction: strain analysis superior to wall thickening analysis in discriminating infarct from remote myocardium," J Am Coll Cardiol, vol. 37, pp. 808-17, Mar 12001.

[10] C. C. Moore, C. H. Lugo-Olivieri, E. R. McVeigh, and E. A. Zerhouni, "Three-dimensional systolic strain patterns in the normal human left ventricle: characterization with tagged MR imaging," Radiology, vol. 214, pp. 453-66, Feb 2000.

[11] A. C. Guyton and J. E. Hall, Textbook of medical physiology, 10th ed. Philadelphia: Saunders, 2000.

[12] P. A. Helm, H. J. Tseng, L. Younes, E. R. McVeigh, and R. L. Winslow, "Ex vivo 3D diffusion tensor imaging and quantification of cardiac laminar structure," Magn Reson Med, vol. 54, pp. 850-9, Oct 2005.

[13] S. Dokos, B. H. Smaill, A. A. Young, and I. J. LeGrice, "Shear properties of passive ventricular myocardium," Am J Physiol Heart Circ Physiol, vol. 283, pp. H2650-9, Dec 2002.

[14] F. Torrent Guasp, "[On cardiac morphology and functionalism. I.]," Rev Esp Cardiol, vol. 19, pp. 48-55, Jan

1966.

[15] F. Torrent-Guasp, "La mecanica agonista-antagonista de los segmentos descendente y ascendente de la banda miocardica ventricular.," Rev Esp Cardiol., vol. 54, pp. 1091-1102, 2001.

[16] F. Torrent-Guasp, M. J. Kocica, A. Corno, M. Komeda, J. Cox, A. Flotats, M. Ballester-Rodes, and F. Carreras-Costa, "Systolic ventricular filling," Eur J Cardiothorac Surg, vol. 25, pp. 376-86, Mar 2004.

[17] D. D. Streeter, Jr., "Gross morphology and fiber geometry of the heart," in Handbook of Physiology. The Cardiovascular ystem. TheHeart. vol. 1 Bethesda: Am. Physiol. Soc., 1979, pp. 61-112. 